\title{
A multiplatform metabolomic approach to characterize fecal signatures of negative postnatal events in chicks: a pilot study
}

Stéphane Beauclercq ${ }^{1}$, Antoine Lefèvre ${ }^{2}$, Frédéric Montigny², Anne Collin ${ }^{1}$, Sophie Tesseraud ${ }^{1}$, Christine Leterrier ${ }^{3}$, Patrick Emond ${ }^{2,4,5}$ and Laurence A. Guilloteau ${ }^{1 *}$ (D)

\begin{abstract}
Background: Negative experiences in early life can induce long-lasting effects on the welfare, health, and performance of farm animals. A delayed placement of chicks in rearing houses has negative effects on their performance, and results in fecal-specific odors detectable by rats. Based on this observation, the volatile organic compounds (VOCs) and metabolites from the feces of 12-day-old chickens were screened for early markers of response to negative events using gas-chromatography and liquid-chromatography coupled with mass spectrometry (GC-MS, LC-HRMS).
\end{abstract}

Results: The low reproducibility of solid-phase micro-extraction of the VOCs followed by GC-MS was not suitable for marker discovery, in contrast to liquid extraction of metabolites from freeze-dried feces followed by GC-MS or LC-HRMS analysis. Therefore, the fecal metabolome from 12-day-old chicks having experienced a normal or delayed placement were recorded by GC-MS and LC-HRMS in two genotypes from two experiments. From both experiments, 25 and 35 metabolites, respectively explaining $81 \%$ and $45 \%$ of the difference between delayed and control chickens, were identified by orthogonal partial least-squares discriminant analysis from LC-HRMS and GC-MS profiling.

Conclusion: The sets of molecules identified will be useful to better understand the chicks' response to negative events over time and will contribute to define stress or welfare biomarkers.

Keywords: Biomarker, Chick, Feces, GC-MS, LC-HRMS, Negative postnatal event

\section{Background}

The perinatal period is a critical period for livestock animals. Recent studies have highlighted that the stress experienced during the perinatal period has long-term consequences on the health and the welfare of broilers [1]. After hatching, the chicks can be exposed to various factors such as temperature variations, confinement, and movement or vibrations during transportation without access to water and feed for up to $3 \mathrm{~d}$. This postnatal environment can influence the behavior of adult broilers [2], their performance (i.e. body weight, feed conversion ratio, Pectoralis major muscle weight) $[3,4]$, their susceptibility to diseases that could lead to death [5], which impacts animal welfare with economic and social issues

\footnotetext{
* Correspondence: laurence.guilloteau@inra.fr

${ }^{1}$ BOA, INRA, Université de Tours, 37380 Nouzilly, France

Full list of author information is available at the end of the article
}

to be considered for the sustainability of poultry rearing industry. Therefore, the early detection of persisting responses to stressful events has become a major challenge for appropriate animal health and welfare management. The terminology of stressful or negative events was used to qualify the postnatal treatment lived by chicks whether it resulted in long lasting effects or not.

Easy, non-invasive methods to detect a persisting response to stressful events are currently lacking in poultry. However, new perspectives are opening up with the development of research on body odor related to physiological status or health issues, and metabolomics research more generally. For instance, the analysis of volatile organic compounds (VOC) can help, among others, in the diagnosis of colorectal cancer (blood, urine, feces, and breath) [6], irritable bowel syndrome (breath) [7], psychological stress (skin) in humans [8] as

(c) The Author(s). 2019 Open Access This article is distributed under the terms of the Creative Commons Attribution 4.0 International License (http://creativecommons.org/licenses/by/4.0/), which permits unrestricted use, distribution, and reproduction in any medium, provided you give appropriate credit to the original author(s) and the source, provide a link to the Creative Commons license, and indicate if changes were made. The Creative Commons Public Domain Dedication waiver (http://creativecommons.org/publicdomain/zero/1.0/) applies to the data made available in this article, unless otherwise stated. 
well as Campylobacter (feces) infection in chickens [9]. The analysis of small polar or lipophilic non-volatile molecules by nuclear magnetic resonance or mass spectrometry metabolomics is another powerful means to non-invasively detect subtle phenotypic changes, which are critical for farm management, but this method has rarely been applied in livestock studies [10]. However, some advances have recently been achieved in chickens to predict or to have a better understanding of quality or digestive physiology issues using blood, muscle, and digestive content metabolomes [11-13].

Exposures to stressful situations alter the gut microbiota composition and impact the composition of the feces as well as their odor, which in the case of rodents can be distinguished by conspecifics and cause avoidance $[14,15]$. The smelling ability of rats holds promise as a tool for detecting stress or health issues and has inspired the development of electronic noses. For example, trained rats are at least as sensitive as the conventional Ziehl-Neelsen stain for detecting tuberculosis in sputum [16]. Metabolomes are currently not exploited for stress diagnosis in mammals or birds, with the exception of measuring glucocorticoid metabolites in blood or feces $[17,18]$, despite their usefulness in assessing the consequences of stressful events on the global metabolism.

An experimental model reproducing adverse perinatal conditions in chicks was previously developed and the potential of rats to distinguish exposed chicks based on their fecal odors was tested with success [14]. In fact, Bombail et al. highlighted a behavior change in the rats in the presence of chicken feces collected $12 \mathrm{~d}$ after the birds had been exposed to a negative postnatal experience, which may confirm the existence of stress-specific odors in poultry [14].

The aim of the present pilot study was thus to evaluate the potential of fecal VOC and metabolites in chicks at 12 $\mathrm{d}$ as early signatures of a negative postnatal experience. Due to the low reproducibility of VOC capture, the fecal metabolome acquired by GC-MS and LC-HRMS containing fewer volatile molecules was chosen for study. The chick fecal metabolomes (i.e. fecal matter and urine) were studied in two different genotypes of broilers to identify a potential typical metabolic signature that could be associated with a negative postnatal experience. This is part of a first attempt to identify potential biomarkers of value for early signatures of this experience.

\section{Methods}

All chemicals were bought from Sigma Aldrich (SaintQuentin Fallavier, France) unless otherwise specified.

\section{Birds and sample collection}

Chicks randomly selected were either directly placed in the experimental rearing facility after their withdrawal from the incubator (control group or $\mathrm{C}$ ) or subjected to a delay period before their placement (delayed group or D) to be compared for the analysis of the consequences of this negative experience as described previously [19]. The delayed group was deprived of feed and water and put in transportation boxes under irregular movement and variable room temperature: $32^{\circ} \mathrm{C}(30 \mathrm{~min}), 21^{\circ} \mathrm{C}(90$ $\min ), 32{ }^{\circ} \mathrm{C}(30 \mathrm{~min})$, and then at $21^{\circ} \mathrm{C}$ with alternated cycles of box movement (M) and immobility (I) for $24 \mathrm{~h}$ after hatching. One cycle was $45 \mathrm{~min}(\mathrm{M}), 15 \mathrm{~min}$ (I), 30 $\min (\mathrm{M}), 30 \mathrm{~min}(\mathrm{I})$ [19]. The $\mathrm{D}$ and $\mathrm{C}$ chicks were thereafter reared together in separated pens $(1 \mathrm{~m} \times 3 \mathrm{~m})$ in the same room at the Pôle d'Expérimentation Animale de Tours (PEAT) (INRA Centre Val de Loire, France) in floor pens in standard temperature and light conditions $(16 \mathrm{~h}$ light and $8 \mathrm{~h}$ darkness) with ad libitum access to water and feed (metabolizable energy $=12.8 \mathrm{MJ} / \mathrm{kg}$, crude protein $=22 \%$ ).

The chicks (males and females) originated from Hubbard Classic genotype (Quintin, France) in experiment 1 and from the Ross 308 (Aviagen, Angers, France) genotype in experiment 2, which are both standard fast-growing broiler lines [20]. The Hubbard Classic cohort was composed of 13 males (6 delayed and 7 controls) and 12 females ( 6 delayed and 6 controls), and the Ross 308 cohort included 11 males (6 delayed and 5 controls) and 12 females ( 6 delayed and 6 controls). The feces (including urine) of control $(C)$ and delayed fed (D) chicks were sampled individually within sterile petri dishes in clean buckets at $12 \mathrm{~d}$ of age (11 d after delayed placement), $2 \mathrm{~h}$ after lighting was on, then packaged in closed $2 \mathrm{~mL}$ glass vials and frozen $\left(-80^{\circ} \mathrm{C}\right)$ before VOC or fecal metabolite extraction. Chicken body weight was measured at $12 \mathrm{~d}$ of age.

\section{Fecal volatilome}

The VOC extraction parameters were optimized using a pool of feces from 180 chicks from the Hubbard Classic control group.

\section{Solid-phase microextraction (SPME)}

Headspace SPME [21] was performed on $400 \mathrm{mg}$ of fresh feces enclosed in a 2-mL glass vial. The extraction protocol was optimized by testing 4 extraction temperatures $\left(20^{\circ} \mathrm{C}, 40^{\circ} \mathrm{C}, 60^{\circ} \mathrm{C}\right.$, and $\left.80^{\circ} \mathrm{C}\right)$ and 3 exposure times (30 min, $1 \mathrm{~h}, 2 \mathrm{~h}$ ) using $100 \mu \mathrm{m}$ polydimethylsiloxane (PDMS) non-bonded fiber. Furthermore, 5 other SPME fibers $(30 \mu \mathrm{m}$ PDMS, $7 \mu \mathrm{m}$ PDMS, $75 \mu \mathrm{m}$ carboxen (CAR)/PDMS, $65 \mu \mathrm{m}$ PDMS/divinylbenzene, and $85 \mu \mathrm{m}$ polyacrylate) from Supelco (Bellefonte, PA) were tested for the maximum number of VOC recovered. The optimal extraction parameters were $1 \mathrm{~h}$ at $60^{\circ} \mathrm{C}$ under magnetic agitation using the $85 \mu \mathrm{m}$ polyacrylate fiber, which is designed for capturing polar semi-volatile 
molecules (MW 80-300). The analytical variability of VOC extraction and GC-MS analysis was assessed by calculating the variation of total feature intensities among 3 replicate samples.

\section{GC-MS analyses}

A Shimadzu GC-MS system (Kyoto, Japan) was used. It was composed of an AOC-20S auto-sampler, an AOC-20i auto-injector, a gas chromatograph 2010, and a QP-2010-Plus mass spectrometer. The VOC samples were desorbed from the fibers in the injection port at $250{ }^{\circ} \mathrm{C}$ and separated on a Zebron capillary ZB-5, 15 $\mathrm{m} \times 0.25 \mathrm{~mm}$ i.d., $0.25 \mu \mathrm{m}$ film thickness $\mathrm{GC}$ column (Phenomenex, Torrance, CA). The oven temperature was set at $50^{\circ} \mathrm{C}$ for $1 \mathrm{~min}$, ramped to $250^{\circ} \mathrm{C}$ at $10^{\circ} \mathrm{C} /$ min and then held for $5 \mathrm{~min}$. Helium was used as the carrier gas and set at $1.2 \mathrm{~mL} / \mathrm{min}$. The ion source and interface temperature were $200{ }^{\circ} \mathrm{C}$ and $250{ }^{\circ} \mathrm{C}$, respectively. The mass spectra of all GC peaks were generated by electron impact (EI) at $70 \mathrm{eV}$ and recorded in a positive total ion monitoring mode scanning the $35-500 \mathrm{~m} / \mathrm{z}$ range (event time $=0.2$ ).

\section{Fecal metabolome}

Specific preanalytical protocols were adapted from Diémé et al. including metabolome extraction followed by chemical derivatization for GC-MS analysis [22].

\section{GC-MS}

Ten mg of freeze-dried chick feces were put into $2 \mathrm{~mL}$ acidified salt water $(120 \mathrm{mmol} / \mathrm{L} \mathrm{HCl}, 0.9 \% \mathrm{NaCl})$, followed by vortexing and centrifugation $\left(3,000 \times g, 20^{\circ} \mathrm{C}\right)$. The metabolites were further extracted from the mix by 2 $\mathrm{mL}$ of ethyl acetate. The organic phase was recovered and evaporated in a SpeedVac (Thermo Fisher Scientific, Waltham, MA) at room temperature. Each sample was derivatized by the addition of $70 \mu \mathrm{L}$ of a mixture of N,O-bistrifluoroacetamide (BSTFA) and trimethylchlorosilane (TMCS; BSTFA/TMCS: 99/1), and $30 \mu \mathrm{L}$ of acetonitrile for $40 \mathrm{~min}$ at $80^{\circ} \mathrm{C}$ in a sand bath. A volume of $1 \mu \mathrm{L}$ of the derivatized mixture was injected in the GC-MS system previously described with the oven temperature set at $80^{\circ} \mathrm{C}$ for $3 \mathrm{~min}$, ramped to $250^{\circ} \mathrm{C}$ at $5{ }^{\circ} \mathrm{C} / \mathrm{min}$ and then held for $25 \mathrm{~min}$. The carrier gas $(\mathrm{He})$ flow was set at $1.0 \mathrm{~mL} / \mathrm{min}$. The instrumental stability and extraction reproducibility were evaluated by multiple injections $(n=6)$ of a quality control (QC) sample obtained from the extraction of $10 \mathrm{mg}$ from a pool of all samples analyzed.

\section{LC-HRMS}

Three $\mathrm{mg}$ of freeze-dried chick feces were put into $500 \mu \mathrm{L}$ of a methanol/water mix $(8: 2)$ and vortexed for 2 $\min$. The mixes were centrifuged $\left(15,000 \times g, 15 \mathrm{~min}, 4^{\circ} \mathrm{C}\right)$ to sediment solid particles, then $450 \mu \mathrm{L}$ of the supernatant was recovered and put in glass tubes for further solvent evaporation in a SpeedVac at room temperature. The residues were then reconstituted with $200 \mu \mathrm{L}$ methanol/water (1:1) followed by centrifugation $\left(20,000 \times g, 10 \mathrm{~min}, 4^{\circ} \mathrm{C}\right)$ before Ultra-High-Performance Liquid Chromatography (UHPLC) separation and mass spectrometry analysis. LC-HRMS analysis was performed on a UHPLC Ultimate 3000 system (Dionex, Sunnyvale, CA), coupled to a Q-Exactive mass spectrometer (Thermo Fisher Scientific) and operated in positive (ESI+) and negative (ESI-) ionization modes. Chromatography was carried out with a $1.7-\mu \mathrm{m} \mathrm{XB}-\mathrm{C} 18(150 \mathrm{~mm} \times 2.10 \mathrm{~mm}, 100 \AA)$ UHPLC column (Kinetex, Phenomenex, Torrance, $\mathrm{CA}$ ) heated at $40^{\circ} \mathrm{C}$. The solvent system comprised mobile phase A [water $+0.1 \%(v / v)$ formic acid], and mobile phase B [methanol $+0.1 \%(v / v)$ formic acid]; the gradient operated at a flow rate of $0.4 \mathrm{~mL} / \mathrm{min}$ over a run time of $24 \mathrm{~min}$. The multistep gradient was programmed as follows: 0 $1.5 \mathrm{~min}, 32-45 \% \mathrm{~A} ; 1.5-5 \mathrm{~min}, 45-52 \%$ A; $5-8 \mathrm{~min}$, $52-58 \%$ A; 8-11 min, 58-66\% A; $11-14 \mathrm{~min}, 66-70 \%$ A; $14-18$ min, $70-75 \%$ A; $18-21$ min, $75-97 \%$ A; $21-$ $24 \mathrm{~min}, 97 \% \mathrm{~A}$. The autosampler (Ultimate WPS-3000 UHPLC system, Dionex, Sunnyvale, CA) temperature was set at $4{ }^{\circ} \mathrm{C}$, and the injection volume for each sample was $5 \mu \mathrm{L}$. Heated ESI source parameters were a spray voltage of $3.5 \mathrm{kV}$, capillary temperature of $350^{\circ} \mathrm{C}$, heater temperature of $250^{\circ} \mathrm{C}$, sheath gas flow of 35 arbitrary units (AU), auxiliary gas flow of $10 \mathrm{AU}$, spare gas flow of $1 \mathrm{AU}$, and tube lens voltage of $60 \mathrm{~V}$ for $\mathrm{C} 18$. During the full-scan acquisition, which ranged from 58 to $870 \mathrm{~m} / z$, the instrument operated at 70,000 resolution $(m / z=400)$, with an automatic gain control target of $1 \times 10^{6}$ charges and a maximum injection time of $250 \mathrm{~ms}$. The instrumental stability was evaluated by multiple injections $(n=6)$ of a QC sample obtained from a pool of $10 \mu \mathrm{L}$ of all samples analyzed. This $\mathrm{QC}$ sample was injected once at the beginning of the analysis, every 10 sample injections, and at the end of the run.

\section{Data processing and spectral assignment GC-MS}

Each chromatogram obtained was processed for smoothing, library matching, and area calculation using the GC-MS Solution Postrun Analysis software (Shimadzu, Japan) as described in Emond et al. [23]. To minimize process errors, each integrated peak was manually checked for each sample and features with greater than $30 \%$ variability in QC samples were rejected [24]. Compounds were identified from their electron impact mass spectra by comparison to our in-house library ( $250 \mathrm{~mol}$ ecules) combined with the NIST spectral mass library (NIST 05), HMDB [25], and ChemSpider. This annotation reached level 2 on the scale of confidence in 
metabolite identification, as defined by the Chemical Analysis Working Group of the Metabolomics Standards Initiative [26]. The feature areas were normalized by dividing each one by the total area of the chromatogram and by the weight of each dried sample.

\section{LC-HRMS}

A library of standard compounds (Mass Spectroscopy Metabolite Library of standards MS $\mathrm{ML}^{\circ}$, IROA technologies) was analyzed with the same gradient of mobile phases and in the same conditions as those used to analyze feces samples. The annotation of selected features was validated from retention time, and high-resolution mass molecular. Targeted molecules (367 molecules detected in ESI+, 255 in ESI-) were selected and integrated into Xcalibur 2.2 (Thermo Fisher Scientific, San Jose, CA) as described by Bitar et al. [27]. This annotation reached level 1 on the scale of confidence in metabolite identification [26]. Each peak area was normalized to the total peaks area of each chromatogram and by the weight of each dried sample. Peaks with greater than $30 \%$ variability in QC samples were rejected as unsuitable for further investigation.

\section{Chemometry and statistical analysis}

The effects of the delayed placement on body weight were analysed by ANOVA after having checked the normality of data distribution (XLSTAT software, Addinsoft, Paris, France).

Principal Component Analyses (PCA) were performed on the variance unit scaled data sets from the GC-MS and LC-HRMS fecal metabolome analyses as an exploratory unsupervised analysis. Individuals outside of the 95\% confidence interval of the Hotelling's T-square in the GC-MS and LC-HRMS experiments were considered to be outliers and excluded from the subsequent analyses [28].

An orthogonal projection to latent structures discriminant analysis (OPLS-DA) was performed using the SIMCA 13 Software (version 13.0, Umetrics, Umeå, Sweden) on the two data sets (i.e., GC-MS, LC-HRMS). All data were scaled to unit variance to maximize the separation between the delayed and control groups. OPLS-DA is a method of supervised classification that predicts the categorical factor $Y$ (C or D group) by explanatory quantitative variables $X$ (metabolites). OPLS-DA modeling was chosen instead of partial least squares discriminant analysis (PLS-DA) for its inclusion of orthogonal components containing variations that do not contribute to the discrimination between the control and delayed groups. Variation in the spectral data $X$ is divided into one predictive component containing variations correlated with the class identifier $Y$ and single or multiple orthogonal components containing variations orthogonal to the predictive component that do not contribute to discrimination between the defined groups [29]. The minimum number of features needed for optimal classification of the OPLS-DA models was determined by iteratively excluding the variables with low regression coefficients and wide confidence intervals derived from jackknifing combined with low variable importance in the projection (VIP) until maximum improvement of the quality of the models. The metabolite with the lowest $P$-value (Welch's means equality $t$-test) was conserved in the model if the same metabolite was detected in the positive and negative ionization mode for LC-HRMS. The model quality was evaluated after 7 -fold cross validation by cumulative $R^{2} Y$ (goodness of fit), cumulative $Q^{2}$ (goodness of prediction), and CV-ANOVA (cross validation-analysis of variance). CV-ANOVA is a diagnostic tool for assessing the reliability of OPLS-DA models; the returned $\mathrm{P}$-value is indicative of the statistical significance of the fitted model [30]. The contribution of each predictor in the model was evaluated through the variable score contribution (i.e. the differences, in scaled units, for all the terms in the model, between the outlying observation and the normal observation, multiplied by the absolute value of the normalized weight) and the importance in the model (VIP). Subsequently, the OPLS-DA models, as described above, were fitted on the GC-MS and LC-HRMS data sets, considering the genotypes (i.e. Hubbard Classic, Ross 308) independently.

The biochemical information about the metabolites retained in the OPLS-DA models that was used to develop the discussion was partly extracted from the HMDB database [25] and KEGG [31].

\section{Results}

\section{Effect of delayed placement on chicken growth}

The body weight of chicks at $12 \mathrm{~d}$ of age was significantly impacted by the genotype $(P=0.001)$ and by the delayed placement in rearing facilities for both genotypes $(P<0.0001)$ (Fig. 1$)$. There were significant reductions by $9.7 \%$ and $9.8 \%$ of the average body weight between the control (C) and the delayed (D) for Hubbard Classic and Ross 308 genotypes, respectively. Those differences were conserved until slaughter (34 d) as previously reported [19]. In these experimental conditions, the delayed placement did not affect the welfare and the prevalence of health disorders [19]. The feces from all the animals were sampled individually for fecal volatilome and metabolome analyses at $12 \mathrm{~d}$ of age for the control and delayed chick groups.

\section{Fecal volatilome}

The extraction time and temperature were tested on a pool of feces from the Hubbard Classic genotype chicks using $100 \mu \mathrm{m}$ polydimethylsiloxane SPME fiber designed 


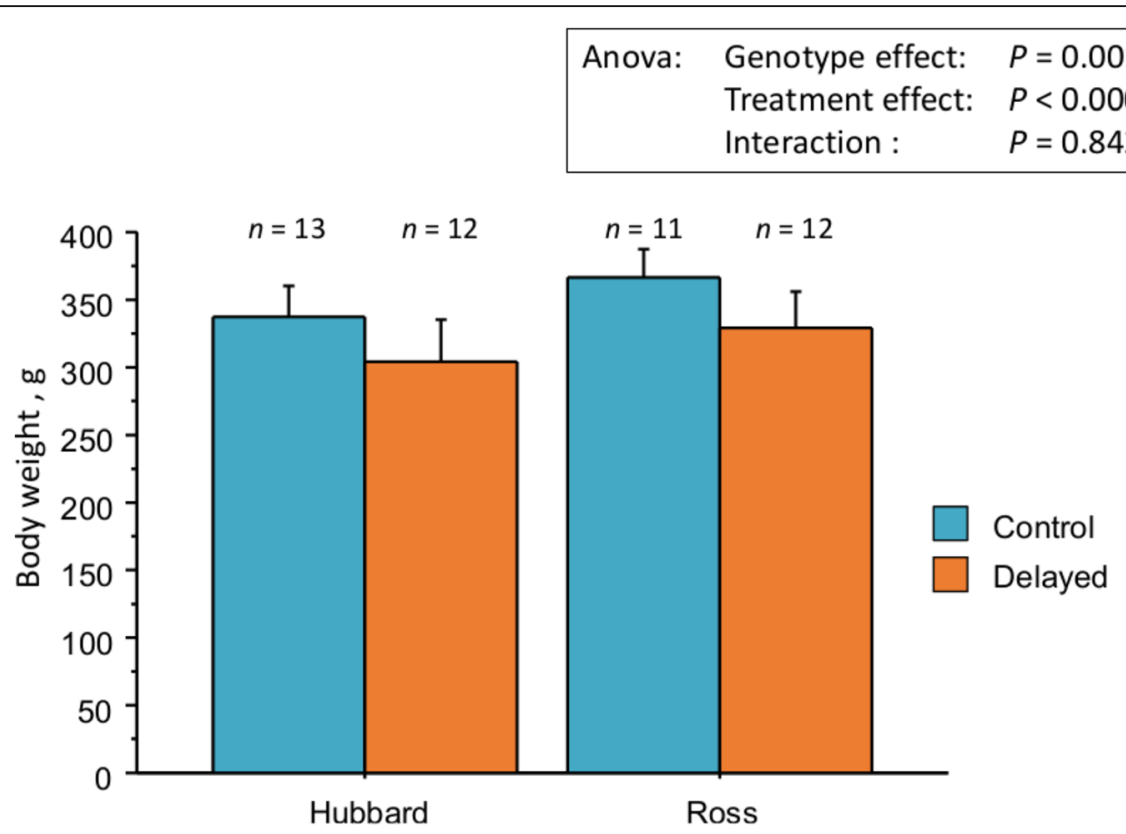

Fig. 1 Effect of delayed placement on chicken body weight. The histograms indicated the mean and standard deviation of the delayed (D) and the control (C) groups at $12 \mathrm{~d}$ of age. The statistical analysis of the different effects is indicated on the figure

to capture volatile molecules (red, MW 60-275). It appeared from those tests that the capture of VOCs in the head space at $60^{\circ} \mathrm{C}$ for $1 \mathrm{~h}$ resulted in a GC-MS chromatogram containing 49 peaks. Using the same extraction conditions, the number of peaks was increased to 56 and 93 using $65 \mu \mathrm{m}$ polydimethylsiloxane/divinylbenzene fiber (blue) and $85 \mu \mathrm{m}$ polyacrylate fiber (white), respectively. Those fibers are designed to capture volatiles, amines, and nitro-aromatic compounds (MW 50-300), and polar semi-volatile molecules (MW 80-300), respectively. It appeared from those developments that the best experimental conditions for capturing VOCs were $1 \mathrm{~h}$ at $60^{\circ} \mathrm{C}$ using the $85 \mu \mathrm{m}$ polyacrylate SPME fiber. The chromatogram acquired using the optimized capture method on 3 replicates of feces from Hubbard Classic control group chicks contained on average 100 features corresponding to around 30 identified molecules (Additional file 1: Table S1). However, the capture was not reproducible because the feature total area variance was $46 \%$ for the 3 replicates. The head space SPME on chick feces was not suitable for quantitative analyses because of lack of reproducibility.

\section{Fecal metabolome}

PCA analyses of the LC-HRMS and GC-MS metabolomics data showed that the QC samples were clustered, which validates the stability of the analysis quality during the analysis campaign (data not shown). The PCA plots also revealed that one sample could be considered to be an outlier (Hubbard Classic, female, delayed) and thus was removed from the subsequent analysis. Furthermore, those PCA highlighted that the genotype of the chicks (i.e. Hubbard Classic and Ross 308) has a higher impact on the fecal metabolome than the sex or the postnatal experience effect (Additional file 1: Figure S1), although both genotypes are selected for rapid growth and lean tissue deposition [20].

A total of 91 and 139 molecules out of the 255 and 367 metabolites present in our in-house data base were detected by LC-HRMS in ESI- and ESI+, respectively, in the Hubbard Classic and Ross 308 chicks. GC-MS, which is more suitable for quantifying volatile non-polar metabolites, allowed the detection of 114 metabolites in both genotypes.

\section{Fecal markers of a previous negative experience}

The LC-HRMS and GC-MS data were fitted to 2 OPLS-DA models, a multivariate supervised classification method aiming to identify 2 sets of metabolites discriminating the $\mathrm{C}$ and $\mathrm{D}$ chick groups and shared by both genotypes.

The consensus model for both genotypes based on the LC-HRMS data (ESI- and ESI+) was composed of 1 predictive and 2 orthogonal components and included 25 metabolites. This model explained $81 \%$ of the differences between the 2 groups $\left(R^{2} Y_{\text {cum }}\right)$ and clearly separated the delayed and control chicks (Fig. 2). The predictive ability of this model $\left(\mathrm{Q}^{2}\right.$ cum $)$ and its CV-ANOVA were 0.73 and $6.78 \times 10^{-10}$, respectively. The consensus model based on GC-MS data (1 predictive +1 orthogonal component) 
containing 35 metabolites was less explicative $\left(R^{2} Y_{\text {cum }}=\right.$ $45 \%)$ and predictive $\left(\mathrm{Q}_{\text {cum }}^{2}=0.2, \mathrm{CV}\right.$-ANOVA $\left.=0.053\right)$ than the LC-HRMS model (Fig. 3). The importance of the metabolites in both models (VIP) and their contributions are presented in Figs. 2 and 3. Only one metabolite (ketoleucine) was present in both models.

The OPLS-DA model adjusted to the metabolomics data (LC-HRMS or GC-MS) based on separately-analyzed Hubbard Classic or Ross 308 chick feces had equivalent or better explicative and predictive abilities for the groups and included fewer metabolites (i.e. 21-17) than the consensus models (Additional file 1: Figures S2 and S3). Only one metabolite (succinate) was common to the consensus,
Hubbard Classic, and Ross 308 OPLS-DA models in GC-MS, which may result from different ways to deal with negative experience between the 2 genotypes.

\section{Discussion}

\section{Fecal volatilome}

The setup of SPME extraction conditions is tricky and species-dependent. For example, as reported by Reade et al., the optimal extraction methods are different between human and murine feces samples [32]. To our knowledge only one study of the chicken fecal volatilome has been previously done using frozen feces $\left(60^{\circ} \mathrm{C}, 1 \mathrm{~h}\right.$ capture with carboxen/polydimethylsiloxane SPME fiber),

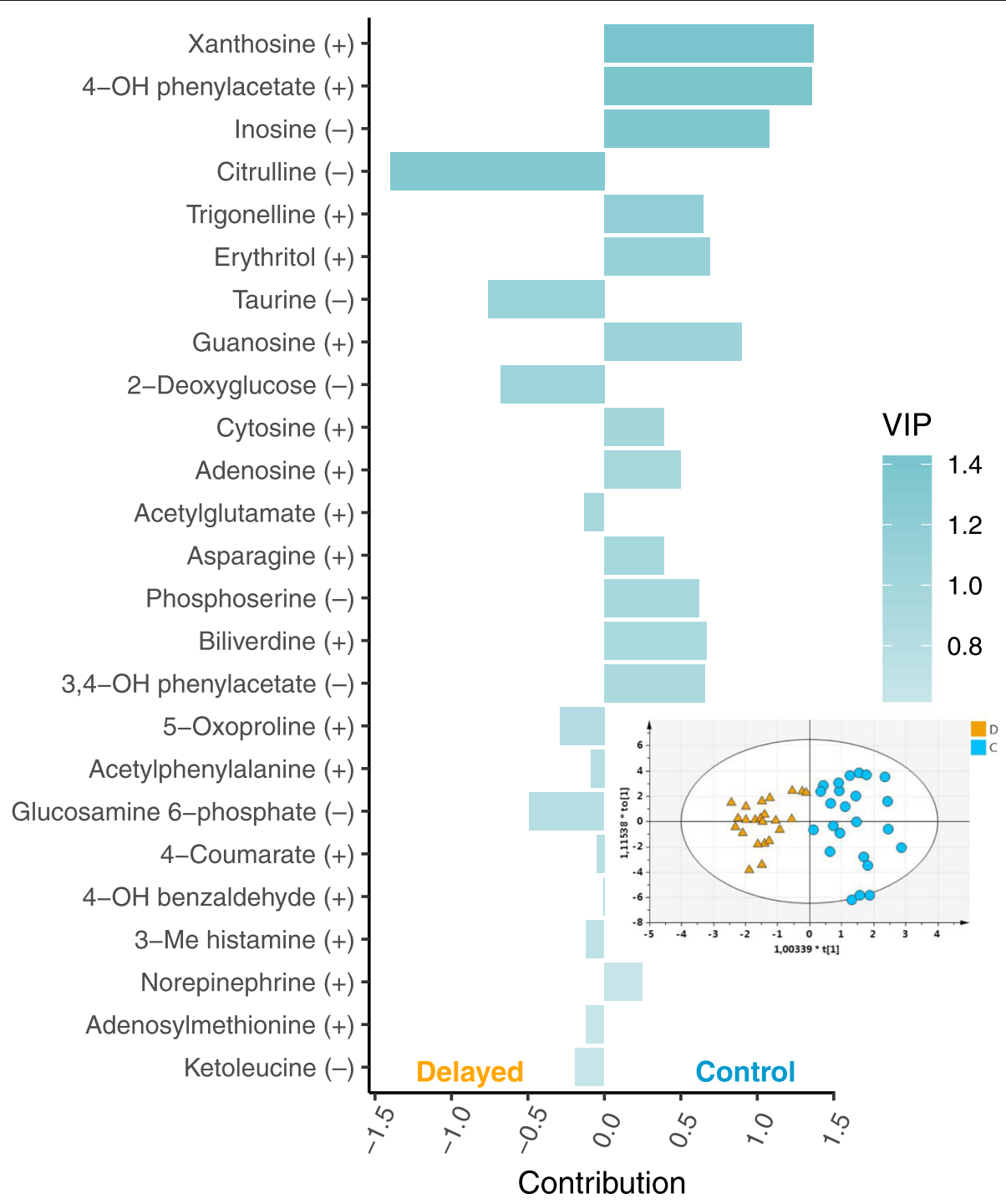

Fig. 2 Discriminant LC-HRMS fecal metabolites (ESI+ and ESI-) and score plot based on OPLS-DA model. In the score plot, the individuals from the delayed (D) and control (C) groups are represented by orange triangles and light blue circles, respectively. The bar plot represents the contributions of the variables (a negative contribution score indicates a contribution of the variable to the delayed group while a positive score is indicative of a contribution to the control group) and their importance in projection (VIP) in the OPLS-DA model. The " + " or " - " signs after the metabolite names correspond to the LC-HRMS ionization mode. The descriptive and predictive performance characteristics of the models are $R^{2} Y_{\text {cum }}=0.81$ and $Q_{\text {cum }}^{2}=0.73$ 


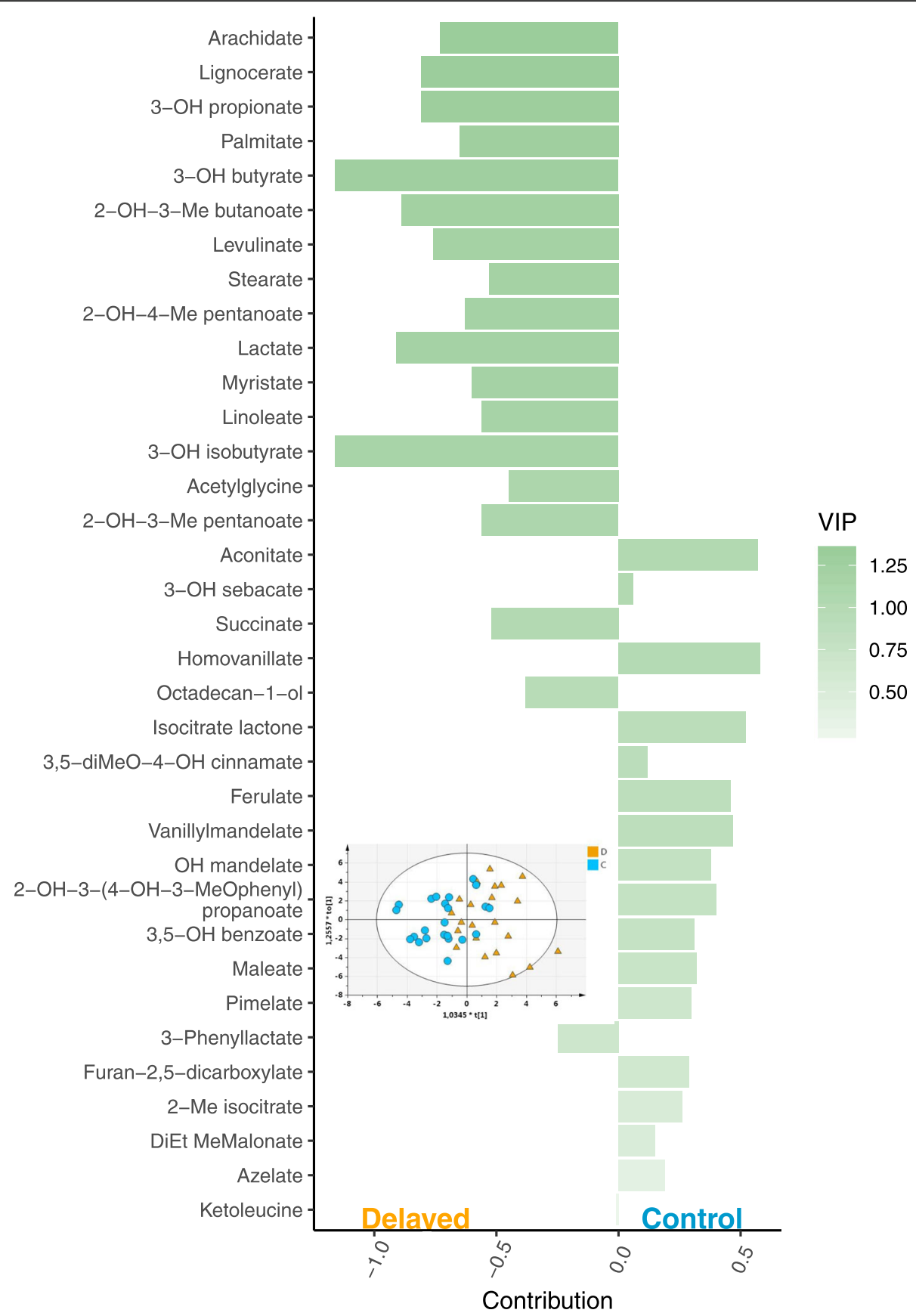

Fig. 3 Discriminant GC-MS fecal metabolites and score plot based on OPLS-DA model. In the score plot, the individuals from the delayed (D) and control (C) groups are represented by orange triangles and light blue circles, respectively. The bar plot represents the contributions of the variables (a negative contribution score indicates a contribution of the variable to the delayed group while a positive score is indicative of a contribution to the control group) and their importance in projection (VIP) in the OPLS-DA model. The descriptive and predictive performance characteristics of the models are $R^{2} Y_{\text {cum }}=0.45$ and $Q_{\text {cum }}^{2}=0.2$

but it did not include reproducibility information [9]. Moreover, the composition of the feces may be different between chickens and chicks. The first step was thus to define the optimal conditions to analyze the fecal volatilome. It appeared from our developments that the best experimental conditions for capturing VOCs were $1 \mathrm{~h}$ at
$60{ }^{\circ} \mathrm{C}$, as proposed in Garner et al., using the $85 \mu \mathrm{m}$ polyacrylate SPME fiber [9]. The head space SPME on chick feces was not suitable for quantitative analyses because of lack of reproducibility. Liquid extraction on freeze-dried feces followed by LC-HRMS or GC-MS was preferred because of higher reproducibility. To illustrate 
this point, the variance of the 250 LC-HRMS features under analysis varied 14\% between the 6 replicates from the pool of feces previously described.

\section{Fecal markers of a previous negative experience}

The OPLS-DA models were a first attempt to characterize the fecal signature of postnatal negative events in chicks. Even if the relative reduction in body weight was similar between the Hubbard Classic and Ross 308 chicks, it appeared that the metabolic profiles related to negative postnatal events were different between the two genotypes (Additional file 1: Figures S2 and S3). The only metabolites (succinate) that was present in consensus and separate models cannot explain the rat behavior changes observed in a previous experiment as it is a non-volatile compound. From the Hubbard Classic (the chicks used in the Bombail et al. rat trial [14]) feces, only 2 volatile compounds were included in the OPLS-DA: cadaverine (LC-HRMS; vapor pressure $=1.0 \pm 0.3 \mathrm{mmHg}$ at $25^{\circ} \mathrm{C}$ ) and guaiacol (GC-MS; vapor pressure $=0.2 \pm 0.4 \mathrm{mmHg}$ at $25^{\circ} \mathrm{C}$ ). Cadaverine is a foul-smelling diamine formed by the decarboxylation of lysine by gut microbiota, while guaiacol is a phenolic product of lignin (grain cell walls [33] or ingested wood shavings from the litter) degradation.

\section{Biological integration of the consensus models}

The consensus OPLS-DA model that was predictive of the postnatal conditions based on the fecal metabolome analyzed by LC-HRMS contained mainly amino acids or their by-products which may come from the diet or the gut microbiota metabolism: citrulline (arginine metabolism), phosphoserine, asparagine, acetylphenylalanine, 5-oxoproline (cyclic sub-product of glutamate), adenosylmethionine (methionine metabolism), and acetylglutamate. Nucleosides and the products of their catabolism were also present in the model (xanthosine, inosine, guanosine, adenosine, cytosine) as well as flavonoid/phenol and other compounds from the diet: 4-hydroxyphenylacetate [34], 4-coumarate (phenylpropanoids related to lignin biosynthesis and flavonoid production), erythritol (polyol), trigonelline (alkaloid produced by the metabolism of niacin present in soybeans [35]; soy is a major component of poultry feeds for protein supply), and 3,4-dihydroxyphenylacetate (phenolic acid formed from fermentation of soy flavonoid). The OPLS-DA model based on GC-MS was less explicative and predictive than the LC-HRMS one. It contained mainly organic acids (3-hydroxypropionate, 3-hydroxybutyrate, 3-hydroxyisobutyrate, lactate, succinate) and fatty acids (palmitate, linoleate, myristate, stearate, arachidate, lignocerate). The extraction using methanol/water for LC-HRMS and ethyl acetate for
GC-MS may explain the difference between the set of metabolites retained in the two OPLS-DA models.

The identification of the metabolic pathways impacted by the negative postnatal experience using fecal metabolomics is rather complex because feces represent the final products of complex interactions involving the gut physiology, the gut immunity, the gut microbiota, the diet, genetics, and the environment [36]. Added to this, urine (in the form of uric acid) and feces are mixed in chickens. For example, the lactate (one of the strongest contributors to the delayed group in GC-MS) could be related to endogenous (cellular energy metabolism) or bacterial metabolism. The OPLS-DA models consensus to both genotypes and fitted to LC-HRMS and GC-MS included metabolites that may be related to an adaptive response to negative events as well as to differences in energy metabolism and microbiota composition between the delayed (D) and control (C) chick groups. Those 3 hypotheses will be discussed in the following paragraphs. The metabolites cited and their spectrum acquisition method as well as their contribution to the OPLS-DA models (i.e. negative values are indicative of a contribution of the metabolite to the $\mathrm{D}$ group and positive values to the $C$ group) will be indicated.

One adrenergic hormone and neurotransmitter from the catecholamine family, norepinephrine (LC, 0.25), contributed less to the fecal metabolome of the delayed group. Norepinephrine is a neurotransmitter produced by the sympathetic nervous system, adrenal glands, and also by some gut bacteria $[37,38]$. It is the primary neurotransmitter found in the gut of most animals and known to be secreted in response to stress, but also to regulate the behavior response to stressful stimuli in chicken [39]. Vanillomandelate (GC, 0.47), a product of norepinephrine catabolism, contributed less to the delayed group as well as 3,4-dihydroxyphenylacetate (LC, $0.66)$ and homovanilate $(\mathrm{GC}, 0.58)$, the intermediate and the final product of dopamine catabolism, respectively. Dopamine, another catecholamine synthetized in the brain and kidneys or released by gut bacteria [37, 38], plays a role in reward processing, but its release is also increased by stress $[40,41]$. The lower level of catecholamine and its metabolites in the feces of the delayed chicks at $12 \mathrm{~d}$ of age suggested that the delayed birds have a lower basal release of catecholamine in standard rearing conditions compared to the control birds, which may be related to an adaptive response over time to a negative postnatal experience. To evaluate this hypothesis, it would be valuable to measure the expression of stress response-related genes and proteins in adrenal glands and the corticosteronemia at the baseline and in response to an acute stress after ACTH treatment [42]. This could be an adaptive process used by the delayed birds to attenuate their stress response as this is 
reported for the domesticated birds compared to their wild counterparts [43]. Furthermore, the contribution of 3 metabolites known for their antioxidant effects $[44,45]$, i.e. citrulline (LC, -1.40$)$, taurine (LC, -0.76$)$, and adenosylmethionine $(\mathrm{LC},-0.12)$ was higher in the delayed groups, also suggesting an adaptive response of the $\mathrm{D}$ chickens to negative postnatal events. The redox balance and the antioxidant status would be interesting to investigate in both delayed and control chickens.

During the first $24 \mathrm{~h}$ post-hatching, the energy metabolism of the 2 groups of chicks ( $D$ and $C$ ) was different and those differences may be conserved during their growth. The blood glucose and triglyceride levels were lower in the D group chicks at day one (unpublished data). The yolk sac is the main source of energy (via fatty acid oxidation) during embryonic development until exogenous feed is given in the brooder house [46]. Therefore, the delayed chicks consumed the rest of the nutrients contained in the internalized yolk sac (mostly fatty acids and proteins) for $24 \mathrm{~h}$ post-hatching while the control group had direct access to exogenous feed rich in carbohydrates (unpublished data). The consensus OPLS-DA models based on the fecal metabolome at 12 $d$ suggested the persistence of a difference in energy metabolism between the 2 groups, at least concerning metabolism in the gut. The major indicator of this difference was the contribution of 3-hydroxybutyrate $(G C,-1.16)$ to the $\mathrm{D}$ group of chicks, which indicated a metabolic activity related to ketogenic amino acid degradation, and lipid $\beta$-oxidation in this group. 3-hydroxybutyrate is an important metabolic substrate for energy production in starvation response [47]. Ketogenic amino acid catabolism can also be illustrated by the contribution of ketoleucine (LC, $-0.19 ; \mathrm{GC},-0.01$ ) to the D chick excreta discrimination in the OPLS-DA model. This suggested a remaining effect of post-hatching starvation or an adaptive effect to lipid-rich substrates $12 \mathrm{~d}$ after the treatment. On the other hand, the digestive utilization of some lipids (medium to long carbon chain) by the $\mathrm{D}$ group was lower as evidenced by the negative contribution of palmitate $(\mathrm{GC},-0.65)$, linoleate $(\mathrm{GC},-0.56)$, myristate $(\mathrm{GC},-0.60)$, stearate $(\mathrm{GC}$, $-0.53)$ and lignocerate $(\mathrm{GC},-0.81)$ to the OPLS-DA models. This may be also related to long-term effects of early food deprivation on hepatic lipid metabolism as reported in early feed restriction program [48]. In contrast, some amino acids contributed less to the fecal metabolome of the D group of chicks, namely asparagine (LC, 0.39 ), and phosphoserine (LC, 0.62 ), suggesting they may have been metabolized and/or used for energy purposes. Interestingly, 3-hydroxyisobutyrate $(\mathrm{GC},-1.16)$, a product of valine catabolism and a good gluconeogenic substrate [49], contributed to the fecal metabolome of the D group of chicks, suggesting enhanced amino acid catabolism that led to glucose production. Therefore, it seems that the group of D chicks exhibits a metabolic adaptation for energy production and utilization compared to the control group and that this adaptation may last more than $10 \mathrm{~d}$ after the $24 \mathrm{~h}$ post-hatch fasting. Beside this, biliverdin (LC, 0.67) contributed less to the fecal metabolome, including urine, of the D group. This compound results from the degradation of heme from hemoglobin in mammals and birds [50,51]. This difference could be related to persisting changes in hepatic or renal function [52], in response to an early negative experience.

The contribution of guanosine (LC, 0.90; purine nucleoside), xanthosine (LC, 1.37), inosine (LC, 1.08), and adenosine (LC, 0.50) was higher in feces from the control chicks compared to the D chicks. Moreover, the xanthosine and the inosine were both in the VIP top 3 of the LC-HRMS OPLS-DA models, which suggests the importance of the pathways related to purine metabolism in the group of control chicks. The cytosine (LC, 0.39) also contributed to the fecal metabolome of the control group. However, it is impossible to determine if those molecules came from endogenous or gut microbiota metabolism. Indeed, some metabolites produced by gut microbiota explained the differences between delayed and control birds: 2-hydroxy-3-methylpentanoate (GC, - 0.56), and 2-hydroxy-4-methylpentanoate (GC, 0.63 ), which are products of isoleucine and leucine catabolism, respectively, by Clostridium difficile for example $[53,54]$, and lactate (GC,- 0.91 ; product of gut microbiota such as Lactobacillus acidophilus in relation with intestinal health [55]). These elements may suggest that the negative postnatal experience (i.e. fasting and stressful conditions) had an impact on the composition of the gut microbiota and the associated metabolome, the next step to investigate.

\section{Conclusion}

The fecal volatilome was not informative due to the lack of reproducibility of the extraction by SPME and injection into the GC-MS in this pilot study, which may be improved by the automatization of the processes. In contrast, liquid extraction of freeze-dried feces followed by LC-HRMS or GC-MS was reproducible, and the OPLSDA model fitted on those data highlights persisting differences in adaptive response, energy metabolism, and microbiota composition for the delayed chicks in response to the negative postnatal experience. Further integrative analyses are needed to characterize these differences in target tissues as brain, adrenal glands, liver for adaptive response and energy metabolism, and gut for microbiota composition. These conclusions bear out the interest of a multiplatform metabolomic approach to characterize the fecal signatures, and possibly of other biological tissues, of response to postnatal negative events. 


\section{Additional file}

Additional file 1: Table S1. Fecal VOCs annotated after headspace solid-phase microextraction (capture $1 \mathrm{~h}$ at $60^{\circ} \mathrm{C}, 85 \mu \mathrm{m}$ polyacrylate fiber) coupled with GC-MS. This volatilome was acquired on a pool of Hubbard control group chick feces. Figure S1. Score plots following principal component analysis for LC-HRMS (a, b, c) and GC-MS (d, e, f) fecal metabolomics. The first components (t [1]) explained 32\% and 37\% of the fecal metabolome variability while the second one (t [2]) explained 10 and $15 \%$ of variability in LC-HRMS and GC-MS, respectively. In the "a" and " $d$ " score plots, Hubbard $(H)$ individuals are represented in turquoise and Ross (R) individuals in purple. The sex of the chicks: male ( $M$, blue) and female (F, red) are represented in the " $b$ " and "e" score plots. The individuals from the delayed group ( $D$, orange triangle) and control group ( $C$, light blue circle) are represented in the " $C$ " and " $f$ " plots. Figure S2. OPLS-DA models adjusted to Hubbard (a) and Ross (b) chick fecal metabolomes at $12 \mathrm{~d}$ of age analyzed by LC-HRMS. The individuals from the delayed group ( $D$, orange triangle) and control group ( $C$, light blue circle) are represented in the score plots at the top of the figure. The fecal metabolites included in the Hubbard and Ross OPLS-DA models are tabulated with their variable importance in projection (VIP) and their contribution in the models. A negative contribution score indicates a contribution of the variable to the delayed group while a positive score is indicative of a contribution to the control group. The performance characteristics of each OPLS-DA model are under the metabolites tables ( $p=$ predictive component, $\mathrm{o}=$ orthogonal component). Figure S3. OPLS-DA models adjusted on Hubbard (a) and Ross (b) chick fecal metabolomes at $12 \mathrm{~d}$ of age analyzed by GC-MS. The individuals from the delayed group ( $D$, orange triangle) and control group (C, light blue circle) are represented in the score plots at the top of the figure. The fecal metabolites included in the Hubbard and Ross OPLS-DA models are tabulated with their variable importance in projection (VIP) and their contribution in the models. A negative contribution score indicates a contribution of the variable to the delayed group while a positive score is indicative of a contribution to the control group. The performance characteristics of each OPLS-DA model are under the metabolites tables ( $p=$ predictive component, $\mathrm{o}=$ orthogonal component). (PDF $1250 \mathrm{~kb}$ )

\section{Abbreviations}

C: Control chicks group; CAR: Carboxen; D: Delayed chicks group; El: Electron impact; ESI: Electrospray ionization; Et: Ethyl; GC-MS: Gaseous chromatography coupled with mass spectrometry; LC-HRMS: Liquid chromatography coupled with high resolution mass spectrometry; Me: Methyl; MeO: Methyloxy; OH: Hydroxy; OPLS-DA: Orthogonal projection to latent structures discriminant analysis; PCA: Principal component analysis; PDMS: Polydimethylsiloxane; PLS-DA: Partial least squares discriminant analysis; $Q^{2}$ cum: Cumulative fraction of the variation of the categorical factor $Y$ predicted by the model; $Q C$ : Quality control; $R^{2} Y_{\text {cum: }}$ : Cumulative fraction of the variation of the categorical factor $Y$ explained by the model; SPME: Solidphase microextraction; UHPLC: Ultra-high performance liquid chromatography; VIP: Variable importance in the projection; VOC: Volatile organic compound

\section{Acknowledgements}

We thank the staff of the Poultry Breeding Facilities (INRA, UE 1295 Pôle d'Expérimentation Avicole de Tours, 37380 Nouzilly, France) for producing the birds, the Avian Research Unit (INRA, UMR Biologie des Oiseaux et Aviculture, 37380 Nouzilly, France), and more particularly Sabine Crochet, Estelle Cailleau-Audouin, and Paul Constantin.

\section{Funding}

This study was supported financially by a "crédits incitatifs" grant from the department of Animal Physiology and Livestock Systems (PHASE) at INRA and by a grant from the Integrated Management of Animal Health metaprogram of INRA for the "GISA-WHELP" project (www.gisa.inra.fr/en).

\section{Availability of data and materials}

The datasets generated and analysed during the current study are available in the MetaboLights repository hosted by the EMBL-EBI, http://www.ebi.ac.uk/ metabolights/MTBLS763).

\section{Authors' contributions}

SB drafted the manuscript and carried out the chemometric analyses. LAG supervised the study. AL performed the LC-HRMS analyses. FM performed the GC-MS analysis. PE supervised the metabolomic analyses. LAG, CL, and AC designed the study and performed experiments and sampling from the birds. ST contributed to the biological integration of the metabolomic data. All authors helped to draft the manuscript and approved the final manuscript.

\section{Ethics approval and consent to participate}

All animal care and experimental procedures needed for this study were conducted in accordance with current European legislation (EU Directive 2010/63/EU) and were approved by the Ethics Committee for Animal Experimentation of Val de Loire [Authorizations No. 01730.02, 2015070815347034v2 (APAFIS\#1082) and 2015072215422490v2 (APAFIS\#1246)]. This ethics committee is registered by the National Committee under the number C2EA 19.

\section{Consent for publication}

All authors approved the final version of the manuscript

\section{Competing interests}

The authors declare that they have no competing interests.

\section{Author details}

${ }^{1}$ BOA, INRA, Université de Tours, 37380 Nouzilly, France. 'Université de Tours, PST Analyse des systèmes biologiques, Tours, France. ${ }^{3}$ PRC, INRA, CNRS, Université de Tours, IFCE, 37380 Nouzilly, France. ${ }^{4}$ UMR 1253, iBrain, Université de Tours, Inserm, Tours, France. ${ }^{5} \mathrm{CHRU}$ de Tours, Service de Médecine Nucléaire In Vitro, Tours, France.

Received: 12 November 2018 Accepted: 10 February 2019 Published online: 09 April 2019

\section{References}

1. Ericsson M, Henriksen R, Bélteky J, Sundman A-S, Shionoya K, Jensen P. Long-term and transgenerational effects of stress experienced during different life phases in chickens (Gallus gallus). PLoS One. 2016;11:e0153879. https://doi.org/10.1371/journal.pone.0153879.

2. Goerlich VC, Nätt D, Elfwing M, Macdonald B, Jensen P. Transgenerational effects of early experience on behavioral, hormonal and gene expression responses to acute stress in the precocial chicken. Horm Behav. 2012;61: 711-8. https://doi.org/10.1016/J.YHBEH.2012.03.006.

3. Bergoug $H$, Guinebretiere $M$, Tong $Q$, Roulston $N$, Romanini CEB, Exadaktylos V, et al. Effect of transportation duration of 1-day-old chicks on postplacement production performances and pododermatitis of broilers up to slaughter age. Poult Sci. 2013;92:3300-9. https://doi.org/10.3382/ps.2013-03118.

4. Bigot K, Mignon-Grasteau S, Picard M, Tesseraud S. Effects of delayed feed intake on body, intestine, and muscle development in neonate broilers. Poult Sci. 2003;82:781-8. https://doi.org/10.1093/ps/82.5.781.

5. Shakeel I, Khan AA, Qureshi S, Adil S, Wani BM, Din MM, et al. Stress levels, mortality, intestinal morphometry and Histomorphology of Chabro broiler birds subjected to varying degrees of post hatch delay in feeding. Pakistan J Biol Sci. 2016;19:331-7. https://doi.org/10.3923/pjbs.2016.331.337.

6. Di Lena M, Porcelli F, Altomare DF. Volatile organic compounds as new biomarkers for colorectal cancer: a review. Color Dis. 2016;18:654-63. https://doi.org/10.1111/codi.13271.

7. Baranska A, Mujagic Z, Smolinska A, Dallinga JW, Jonkers DMAE, Tigchelaar $E F$, et al. Volatile organic compounds in breath as markers for irritable bowel syndrome: a metabolomic approach. Aliment Pharmacol Ther. 2016; 44:45-56. https://doi.org/10.1111/apt.13654.

8. Martin HJ, Turner MA, Bandelow S, Edwards L, Riazanskaia S, Thomas CLP. Volatile organic compound markers of psychological stress in skin: a pilot study. J Breath Res. 2016;10:046012. https://doi.org/10.1088/1752-7155/10/4/ 046012

9. Garner CE, Smith S, Elviss NC, Humphrey TJ, White P, Ratcliffe NM, et al. Identification of Campylobacter infection in chickens from volatile faecal emissions. Biomarkers. 2008;13:413-21. https://doi.org/10.1080/ 13547500801966443

10. Goldansaz SA, Guo AC, Sajed T, Steele MA, Plastow GS, Wishart DS. Livestock metabolomics and the livestock metabolome: a systematic review. PLoS One. 2017;12:e0177675. https://doi.org/10.1371/journal.pone.0177675. 
11. Beauclercq S, Nadal-Desbarats L, Hennequet-Antier C, Collin A, Tesseraud S, Bourin $\mathrm{M}$, et al. Serum and muscle metabolomics for the prediction of ultimate $\mathrm{pH}$, a key factor for chicken-meat quality. J Proteome Res. 2016;15: 1168-78. https://doi.org/10.1021/acs.jproteome.5b01050.

12. Beauclercq S, Nadal-Desbarats L, Hennequet-Antier C, Gabriel I, Tesseraud S, Calenge $F$, et al. Relationships between digestive efficiency and metabolomic profiles of serum and intestinal contents in chickens. Sci Rep. 2018;8:6678. https://doi.org/10.1038/s41598-018-24978-9.

13. Zampiga M, Flees J, Meluzzi A, Dridi S, Sirri F. Application of omics technologies for a deeper insight into quali-quantitative production traits in broiler chickens: a review. J Anim Sci Biotechnol. 2018;9:61. https://doi.org/ 10.1186/s40104-018-0278-5.

14. Bombail V, Barret B, Raynaud A, Jerôme N, Saint-Albin A, Ridder C, et al. In search of stress odours across species: behavioural responses of rats to faeces from chickens and rats subjected to various types of stressful events. Appl Anim Behav Sci. 2017. https://doi.org/10.1016/J.APPLANIM.2017.10.013.

15. Sakuma K, Hayashi S, Yasaka Y, Nishijima H, Funabashi H, Hayashi M, et al. Analysis of odor compounds in feces of mice that were exposed to various stresses during breeding. Exp Anim. 2013;62:101-7. https://doi.org/10.1538/ expanim.62.101.

16. Bijland LR, Bomers MK, Smulders YM. Smelling the diagnosis: a review on the use of scent in diagnosing disease. Neth J Med. 2013;71:300-7.

17. Sheriff MJ, Krebs CJ, Boonstra R. Assessing stress in animal populations: do fecal and plasma glucocorticoids tell the same story? Gen Comp Endocrinol. 2010;166:614-9. https://doi.org/10.1016/J.YGCEN.2009.12.017.

18. Dehnhard M, Schreer A, Krone O, Jewgenow K, Krause M, Grossmann R. Measurement of plasma corticosterone and fecal glucocorticoid metabolites in the chicken (Gallus domesticus), the great cormorant (Phalacrocorax carbo), and the goshawk (Accipiter gentilis). Gen Comp Endocrinol. 2003; 131:345-52. https://doi.org/10.1016/S0016-6480(03)00033-9.

19. Guilloteau L, Collin A, Koch A, Leterrier C. Spontaneous intake and longterm effects of essential oils after a negative postnatal experience in chicks. bioRxiv. 2018:452136. https://doi.org/10.1101/452136.

20. Rahimi S, Esmaeilzadeh L, Karimi Torshizi MA. Comparison of growth performance of six commercial broiler hybrids in Iran. Shiraz Univ. 2006;7: 38-44. https://doi.org/10.22099/IJVR.2006.2661.

21. Vas G, Vékey K. Solid-phase microextraction: a powerful sample preparation tool prior to mass spectrometric analysis. J Mass Spectrom. 2004;39:233-54. https://doi.org/10.1002/jms.606.

22. Diémé B, Lefèvre A, Nadal-Desbarats L, Galineau L, Madji Hounoum B, Montigny $F$, et al. Workflow methodology for rat brain metabolome exploration using NMR, LC-MS and GC-MS analytical platforms. J Pharm Biomed Anal. 2017;142:270-8. https://doi.org/10.1016/j.jpba.2017.03.068.

23. Emond P, Mavel S, Aïdoud N, Nadal-Desbarats L, Montigny F, Bonnet-Brilhault F, et al. GC-MS-based urine metabolic profiling of autism spectrum disorders. Anal Bioanal Chem. 2013;405:5291-300. https://doi.org/10.1007/s00216-013-6934-X.

24. Want EJ, Masson P, Michopoulos F, Wilson ID, Theodoridis G, Plumb RS, et al. Global metabolic profiling of animal and human tissues via UPLC-MS. Nat Protoc. 2012;8:17-32. https://doi.org/10.1038/nprot.2012.135.

25. Wishart DS, Feunang YD, Marcu A, Guo AC, Liang K, Vázquez-Fresno R, et al. HMDB 4.0: the human metabolome database for 2018. Nucleic Acids Res. 2018;46:D608-17. https://doi.org/10.1093/nar/gkx1089.

26. Sumner LW, Amberg A, Barrett $D$, Beale $M H$, Beger $R$, Daykin $C A$, et al. Proposed minimum reporting standards for chemical analysis. Metabolomics. 2007;3:211-21. https://doi.org/10.1007/s11306-007-0082-2.

27. Bitar T, Mavel S, Emond P, Nadal-Desbarats L, Lefèvre A, Mattar H, et al. Identification of metabolic pathway disturbances using multimodal metabolomics in autistic disorders in a middle eastern population. J Pharm Biomed Anal. 2018;152:57-65. https://doi.org/10.1016/J.JPBA.2018.01.007.

28. Jackson JE. A user's guide to principal components. Hoboken: Wiley; 1991. https://doi.org/10.1002/0471725331.

29. Trygg J, Wold S. Orthogonal projections to latent structures (O-PLS). J Chemom. 2002;16:119-28. https://doi.org/10.1002/cem.695.

30. Eriksson L, Trygg J, Wold S. CV-ANOVA for significance testing of PLS and OPLS models. J Chemom. 2008;22:594-600. https://doi.org/10.1002/cem.1187.

31. Kanehisa M, Goto S. KEGG: Kyoto encyclopedia of genes and genomes. Nucleic Acids Res. 2000;28:27-30. https://doi.org/10.1093/nar/28.1.27.

32. Reade S, Mayor A, Aggio R, Khalid T, Pritchard D, Ewer A, et al. Optimisation of sample preparation for direct SPME-GC-MS analysis of murine and human Faecal volatile organic compounds for Metabolomic studies. J Anal Bioanal Tech. 2014;5. https://doi.org/10.4172/2155-9872.1000184.
33. Burton RA, Fincher GB. Evolution and development of cell walls in cereal grains. Front Plant Sci. 2014;5:456. https://doi.org/10.3389/fpls. 2014.00456

34. Rothwell JA, Perez-Jimenez J, Neveu V, Medina-Remón A, M'hiri N, GarcíaLobato P, et al. Phenol-explorer 3.0: a major update of the phenol-explorer database to incorporate data on the effects of food processing on polyphenol content. Database (Oxford). 2013;2013:bat070. https://doi.org/10. 1093/database/bat070.

35. Sánchez-Hernández L, Puchalska P, García-Ruiz C, Crego AL, Marina ML. Determination of Trigonelline in seeds and vegetable oils by capillary electrophoresis as a novel marker for the detection of adulterations in olive oils. J Agric Food Chem. 2010;58:7489-96. https://doi.org/10.1021/jf100550b.

36. Vernocchi P, Del Chierico F, Putignani L. Gut microbiota profiling: metabolomics based approach to unravel compounds affecting human health. Front Microbiol. 2016;7:1144. https://doi.org/10.3389/fmicb.2016.01144.

37. Dai Z, Wu Z, Hang S, Zhu W, Wu G. Amino acid metabolism in intestinal bacteria and its potential implications for mammalian reproduction. Mol Hum Reprod. 2015;21:389-409. https://doi.org/10. 1093/molehr/gav003.

38. Galland L. The gut microbiome and the brain. J Med Food. 2014;17:126172. https://doi.org/10.1089/jmf.2014.7000.

39. Dennis RL. Adrenergic and noradrenergic regulation of poultry behavior and production. Domest Anim Endocrinol. 2016;56:S94-100. https://doi.org/ 10.1016/J.DOMANIEND.2016.02.007.

40. Pruessner JC, Champagne F, Meaney MJ, Dagher A. Dopamine release in response to a psychological stress in humans and its relationship to early life maternal care: a positron emission tomography study using [11C]Raclopride. J Neurosci. 2004;24:2825-31. https://doi.org/10.1523/ JNEUROSCI.3422-03.2004.

41. Cheng $H$, Singleton $P$, Muir W. Social stress in laying hens: differential effect of stress on plasma dopamine concentrations and adrenal function in genetically selected chickens. Poult Sci. 2003;82:192-8. https://doi.org/10. 1093/ps/82.2.192.

42. Bureau C, Hennequet-Antier C, Couty M, Guémené D. Gene array analysis of adrenal glands in broiler chickens following ACTH treatment. BMC Genomics. 2009:10:430. https://doi.org/10.1186/1471-2164-10-430.

43. Løtvedt P, Fallahshahroudi A, Bektic L, Altimiras J, Jensen P. Chicken domestication changes expression of stress-related genes in brain, pituitary and adrenals. Neurobiol Stress. 2017;7:113-21. https://doi.org/10.1016/j.ynstr. 2017.08.002.

44. Wu G. Amino acids: metabolism, functions, and nutrition. Amino Acids. 2009;37:1-17. https://doi.org/10.1007/s00726-009-0269-0.

45. Li Q, Cui J, Fang C, Liu M, Min G, Li L. S-Adenosylmethionine attenuates oxidative stress and Neuroinflammation induced by amyloid- $\beta$ through modulation of glutathione metabolism. J Alzheimers Dis. 2017;58:549-58. https://doi.org/10.3233/JAD-170177.

46. Yadgary L, Cahaner A, Kedar O, Uni Z. Yolk sac nutrient composition and fat uptake in late-term embryos in eggs from young and old broiler breeder hens. Poult Sci. 2010;89:2441-52. https://doi.org/10.3382/ ps.2010-00681.

47. Rojas-Morales P, Tapia E, Pedraza-Chaverri J. $\beta$-Hydroxybutyrate: a signaling metabolite in starvation response? Cell Signal. 2016;28:917-23. https://doi. org/10.1016/J.CELLSIG.2016.04.005.

48. Yang X, Zhuang J, Rao K, Li X, Zhao R. Effect of early feed restriction on hepatic lipid metabolism and expression of lipogenic genes in broiler chickens. Res Vet Sci. 2010;89:438-44. https://doi.org/10.1016/J.RVSC.2010.04.003.

49. Letto J, Brosnan ME, Brosnan JT. Valine metabolism. Gluconeogenesis from 3-hydroxyisobutyrate. Biochem J. 1986;240:909-12. https://doi.org/10.1042/ BJ2400909.

50. Lin G, Himes J, Cornelius C. Bilirubin and biliverdin excretion by the chicken. Am J Physiol Content. 1974;226:881-5. https://doi.org/10.1152/ajplegacy.1974.226.4.881.

51. Hamoud A-R, Weaver L, Stec DE, Hinds TD. Bilirubin in the liver-gut signaling Axis. Trends Endocrinol Metab. 2018;29:140-50. https://doi.org/10. 1016/j.tem.2018.01.002.

52. Harr KE. Clinical chemistry of companion avian species: a review. Vet Clin Pathol. 2002;31:140-51. https://doi.org/10.1111/j.1939-165X.2002.tb00295.x.

53. Kim J, Darley D, Selmer T, Buckel W. Characterization of (R)-2Hydroxyisocaproate dehydrogenase and a family III coenzyme a transferase involved in reduction of L-leucine to Isocaproate by Clostridium difficile. Appl Environ Microbiol. 2006;72:6062-9. https://doi. org/10.1128/AEM.00772-06. 
54. Dai Z-L, Wu G, Zhu W-Y. Amino acid metabolism in intestinal bacteria: links between gut ecology and host health. Front Biosci (Landmark Ed). 2011;16:1768-86 http://www.ncbi.nlm.nih.gov/pubmed/21196263. Accessed 8 Apr 2018.

55. Li Z, Wang W, Liu D, Guo Y. Effects of lactobacillus acidophilus on gut microbiota composition in broilers challenged with Clostridium perfringens. PLoS One. 2017;12:e0188634. https://doi.org/10.1371/journal.pone.0188634.

Ready to submit your research? Choose BMC and benefit from:

- fast, convenient online submission

- thorough peer review by experienced researchers in your field

- rapid publication on acceptance

- support for research data, including large and complex data types

- gold Open Access which fosters wider collaboration and increased citations

- maximum visibility for your research: over $100 \mathrm{M}$ website views per year

At BMC, research is always in progress.

Learn more biomedcentral.com/submissions 